\title{
Correction to: A re-assessment of nickel-doping method in iron isotope analysis on rock samples using multi-collector inductively coupled plasma mass spectrometry
}

\author{
Hongmei Gong ${ }^{1,2} \cdot$ Pengyuan Guo ${ }^{1,2,5} \cdot$ Shuo Chen $^{1,2} \cdot$ Meng Duan $^{1,2} \cdot$ \\ Pu Sun ${ }^{1,2} \cdot$ Xiaohong Wang ${ }^{1,2} \cdot$ Yaoling Niu ${ }^{1,2,3,4}$
}

Published online: 23 January 2020

(C) Science Press and Institute of Geochemistry, CAS and Springer-Verlag GmbH Germany, part of Springer Nature 2020

\section{Correction to: Acta Geochim \\ https://doi.org/10.1007/s11631-019-00392-4}

In the original publication, the vertical coordinate in Fig. 7 is incorrectly published as $\delta^{56} \mathrm{Fe}$ instead of $\delta^{57} \mathrm{Fe}$. The correct Fig. 7 is provided in this correction.
The original article can be found online at https://doi.org/10.1007/ s11631-019-00392-4.

Pengyuan Guo

guopy@qdio.ac.cn

1 Key Laboratory of Marine Geology and Environment, Institute of Oceanology, Chinese Academy of Sciences, Qingdao 266071, China

2 Qingdao National Laboratory for Marine Science and Technology, Qingdao 266061, China

3 Department of Earth Sciences, Durham University, Durham DH1 3LE, UK

4 School of Earth Science and Resources, China University of Geosciences, Beijing 100083, China

5 Present Address: Institute of Oceanology, Chinese Academy of Sciences, Nanhai Road 7, Qingdao 266071, Shandong, China

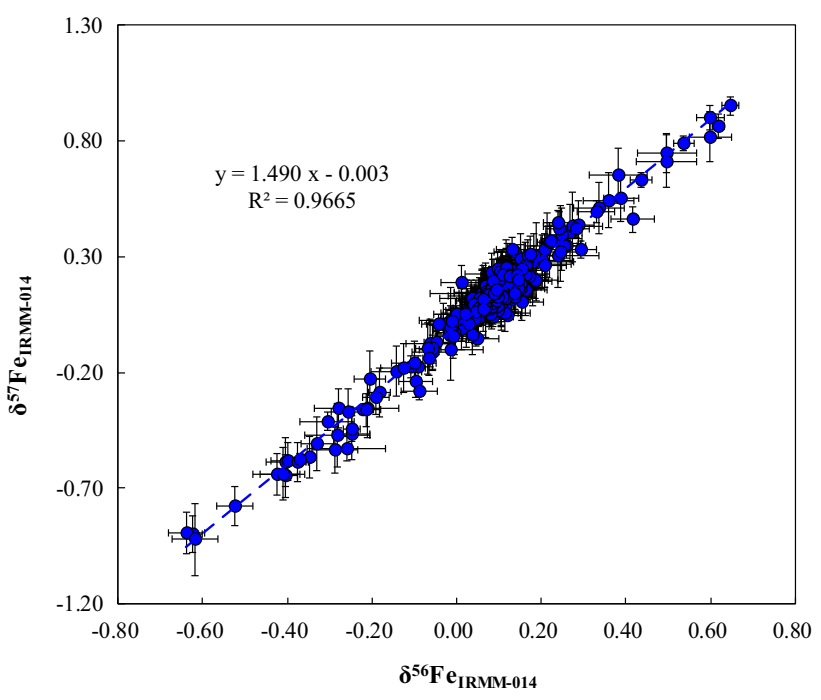

Fig. 7 Iron isotope compositions of various geological samples relative to IRMM-014 analyzed over the period of three months. The gray line represents a linear regression of $\delta^{56} \mathrm{Fe}$ vs. $\delta^{57} \mathrm{Fe}$ with a slope of $1.490 \pm 0.015(\mathrm{SE})\left(\mathrm{R}^{2}=0.9665, \mathrm{~N}=332\right)$. This relationship is statistically consistent with both theoretical predictions of massdependent isotope fractionation (slope of 1.475; Young et al. 2002) and with previously measured isotopic mass-dependent fractionation trends using Nu Plasma (slope of 1.482; Chen et al. 2017a) 